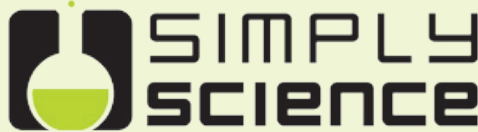

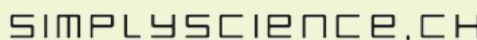

\section{Viehmist und Vogelkot war gestern}

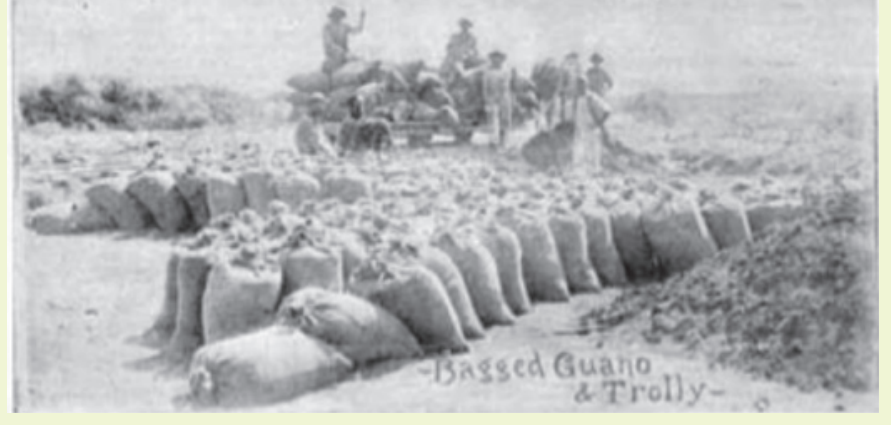

Früher war Vogelkot - Guano genannt - wertvoller Dünger und wurde aus den tropischen Regionen importiert. (Bild: Wikimedia Commons)

Dünger ist das Doping der Landwirtschaft. Anders als beim Sport ist es in der Landwirtschaft aber erlaubt. Lange war Viehmist das einzige Düngemittel. Denn um Dünger künstlich herzustellen, fehlte den Chemikern das nötige Know-how. Erst Mitte des 19. Jahrhunderts konnte man die wichtigste Ausgangschemikalie für Dünger in grossen Mengen produzieren: das Ammoniak.

Schon seit Urzeiten wird der Boden mit nährstoffreichen Substanzen „gefüttert“, um das Wachstum von Pflanzen zu beschleunigen, zum Beispiel weil der Boden zuwenig nährstoffreich ist. In früheren Jahrhunderten musste die Weltbevölkerung oft hungern, weil es an Düngemitteln fehlte. Als es plötzlich möglich war, künstliche Düngemittel herzustellen, konnten die Böden viel intensiver bewirtschaftet werden. Nahrungsmittel waren dadurch in Europa keine Mangelware mehr. Auch deshalb konnten sich viele europäische Länder industriell überhaupt so weit entwickeln.

\section{Wichtige Nährstoffe}

Zum Wachsen braucht eine Pflanze Nährstoffe. Den wichtigsten Nährstoff, Kohlenstoff, holt die Pflanze aus der Luft. Dort kommt Kohlenstoff als Kohlendioxid vor $\left(\mathrm{CO}_{2}\right)$. Es wird von den Pflanzen gebunden und in einem Prozess, der sich Photosynthese nennt, zu Pflanzenbausteinen verarbeitet.

Neben Kohlenstoff benötigt eine Pflanze noch weitere Nährstoffe. Die wichtigsten sind im Dünger enthalten: Stickstoff, Phosphor, Kalium und Magnesium. Viele Böden sind vor allem arm an Stickstoff. Dünger gleicht diesen Mangel aus.

\section{Organisch oder mineralisch?}

Man unterscheidet zwischen organischem Dünger und mineralischem Dünger. Der organische Dünger wird aus tierischen und pflanzlichen Abfällen gemacht. Anders ist der mineralische Dünger, auch Kunstdünger genannt: Er wird direkt nach dem Aufspritzen von den Pflanzen aufgenommen und für ihr Wachstum verwendet. Mineralische Dünger enthalten jede Menge chemische Stickstoffverbindungen.

Um diese Stickstoffverbindungen künstlich herstellen zu können, braucht man Ammoniak. Dieses Gas besteht aus den Elementen Stickstoff und Wasserstoff. Die Herstellung von Ammoniak aus den Gasen Wasserstoff und Stickstoff ist enorm energieintensiv. Es reicht nicht aus, Wasserstoff und Stickstoff in einen Kessel zu werfen, das Ganze zu erhitzen und darauf zu warten, dass dann Ammoniak entsteht.

\section{Hartes Brot: Der Stickstoff}

Das Problem: Das Gas Stickstoff - übrigens Hauptbestandteil unserer Luft - ist chemisch weitestgehend inert. Das bedeutet, Stickstoff reagiert nur mit anderen chemischen Stoffen, wenn es extremen Bedingungen wie sehr hohem Druck und sehr hohen Temperaturen ausgesetzt ist. Der Druck müsste so hoch sein, dass kein Kessel ihm standhalten könnte. Wie man den Stickstoff unter geringerem Druck zum Reagieren drängen könnte, an diesem Problem tüftelten viele Chemiker.

Anfangs des 20. Jahrhunderts hatte der deutsche Chemiker Fritz Haber nach vielem Überlegen und Testen endlich herausgefunden, wie man den Stickstoff unter milderen Bedingungen dazu bringt, mit Wasserstoff zu Ammoniak zu reagieren. Das Verfahren wurde von Carl Bosch technisch umgesetzt. Man verwendet das Haber-Bosch-Verfahren heute auf der ganzen Welt. Jährlich entstehen so etwa 130 Tonnen Ammoniak. Für die Entwicklung dieser Methode erhielt Fritz Haber 1914 den Nobelpreis für Chemie. Für die technische Umsetzung wurde der Preis 1931 an Carl Bosch verliehen.

\section{Das Rätsel hinter Haber-Bosch}

Um den Stickstoff mit Wasserstoff bei relativ niedrigen Temperaturen und einem Druck durchzuführen, bei denen die Kessel nicht platzen, verwendete Fritz Haber einen Katalysator. Bei einem Auto hilft ein Katalysator dabei, dass giftige Autoabgase miteinander zu weniger gesundheitsgefährlichen Stoffen reagieren.

Auch beim Haber-Bosch-Verfahren ermöglicht der Katalysator, dass die Reaktion zwischen Gasen - dem Stickstoff und dem Wasserstoff - bei milderen Temperaturen und Druck ablaufen kann. Im gesamten Haber-Bosch-Verfahren kommen mehrere Katalysatoren zum Einsatz, denn das Verfahren läuft über vier Schritte ab (siehe Bild unten). Welche Stoffe in den einzelnen Schritten miteinander reagieren, das ist darunter erklärt.

Für das Verfahren braucht es eine Menge Energie, ganze 1,4\% des Weltenergieverbrauchs. Um diesen Energieverbrauch zu senken, arbeiten Forscher mit Hochdruck an besseren Katalysatoren. Einen Schritt auf dem Weg dahin hat der deutsche Chemiker Gerhard Ertl gemacht. Er fand heraus, dass die Spaltung des Stickstoffmoleküls $\mathrm{N}_{2}$ in einzelne Stickstoffatome an den Eisenoberflächen des Katalysators den entscheidenden Schritt darstellt. Nach der Spaltung kann der Stickstoff mit Wasserstoff reagieren. Dafür erhielt er erst kürzlich, und zwar im Jahr 2007, den Nobelpreis für Chemie. 


\section{Das Haber-Bosch-Verfahren im Detail:}

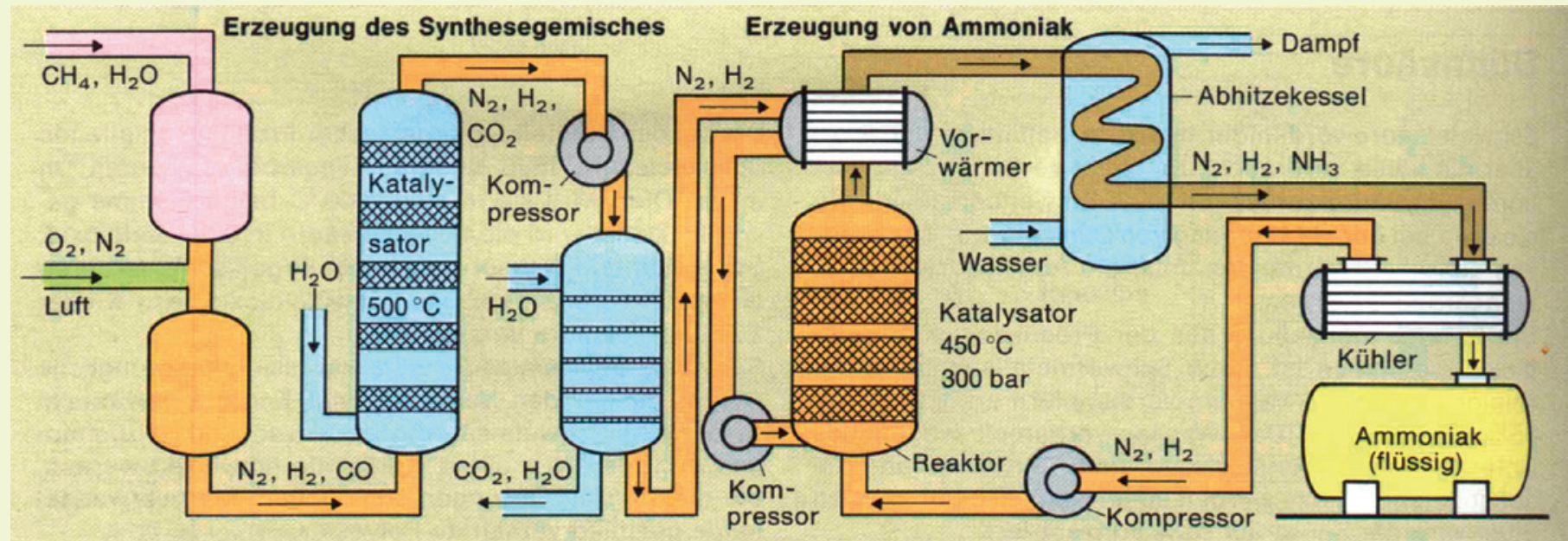

Beim Haber-Bosch-Verfahren gewinnt man zunächst Wasserstoff aus Erdgas und Wasser. Unter hohem Druck und einer Temperatur von $c a .750{ }^{\circ} \mathrm{C}$ wird das Erdgas, welches hauptsächlich aus Methan $\left(\mathrm{CH}_{4}\right)$ besteht, mit Wasserdampf $\left(\mathrm{H}_{2} \mathrm{O}\right)$ vermischt. Ein Katalysator aus Nickeloxid und Aluminiumoxid unterstützt die chemische Reaktion. Es entsteht Kohlenmonoxid $(\mathrm{CO})$ und Wasserstoff $\left(\mathrm{H}_{2}\right)$.

1) $\mathrm{CH}_{4}(\mathrm{~g})+\mathrm{H}_{2} \mathrm{O}(\mathrm{g}) \rightarrow \mathrm{CO}(\mathrm{g})+3 \mathrm{H}_{2}(\mathrm{~g})$

Anschliessend wird Luft beigemischt. Um das Verfahren effizient zu machen, wird das Gemisch weiterhin erhitzt und das übriggebliebene Methan $\left(\mathrm{CH}_{4}\right)$ reagiert mit dem Sauerstoff $\left(\mathrm{O}_{2}\right)$ der zugeführten Luft, es entstehen Kohlenmonoxid (CO) und Wasserstoff $\left(\mathrm{H}_{2}\right)$. Übrigens enthält das Reaktionsgemisch jetzt auch Stickstoff $\left(\mathrm{N}_{2}\right)$. Denn Luft besteht zu 80\% aus Stickstoff. In diesem Schritt passiert aber nichts mit ihm, er bleibt zunächst unberührt im Gemisch.

\section{2) $2 \mathrm{CH}_{4}(\mathrm{~g})+\mathrm{O}_{2}(\mathrm{~g}) \rightarrow 2 \mathrm{CO}+4 \mathrm{H}_{2}(\mathrm{~g})$}

Bei dieser Reaktion entsteht Kohlenmonoxid (CO). Dieses Gas würde die chemische Reaktion zwischen Wasserstoff $\left(\mathrm{H}_{2}\right)$ und Stickstoff $\left(\mathrm{N}_{2}\right)$ stören. Deshalb muss es entfernt werden. Das geschieht mit folgender, sich anschliessender Reaktion, bei der Kohlendioxid $\left(\mathrm{CO}_{2}\right)$ und Wasserstoff $\left(\mathrm{H}_{2}\right)$ entstehen:

3) $\mathrm{CO}(\mathrm{g})+\mathrm{H}_{2} \mathrm{O}(\mathrm{g}) \rightarrow \mathrm{CO}_{2}(\mathrm{~g})+\mathrm{H}_{2}(\mathrm{~g})$

Das entstandene, überschüssige Kohlendioxid (CO) wird durch Waschen des Gasgemischs mit einer wässrigen Kaliumcarbonatlösung entfernt. Im letzten Schritt geschieht nun endlich die Umsetzung von Stickstoff und Wasserstoff zu Ammoniak. Bei $450{ }^{\circ} \mathrm{C}$ und einem Druck von $230-330$ bar reagieren beide Ausgangsgase miteinander. Wichtig auch bei dieser Reaktion: ein Katalysator.
4) $\mathrm{N}_{2}+6 \mathrm{H}_{2}(\mathrm{~g}) \rightarrow 2 \mathrm{NH}_{3}(\mathrm{~g})$

Das entstandene Ammoniakgas wird später mit weiteren Chemikalien zu Kunstdünger verarbeitet.

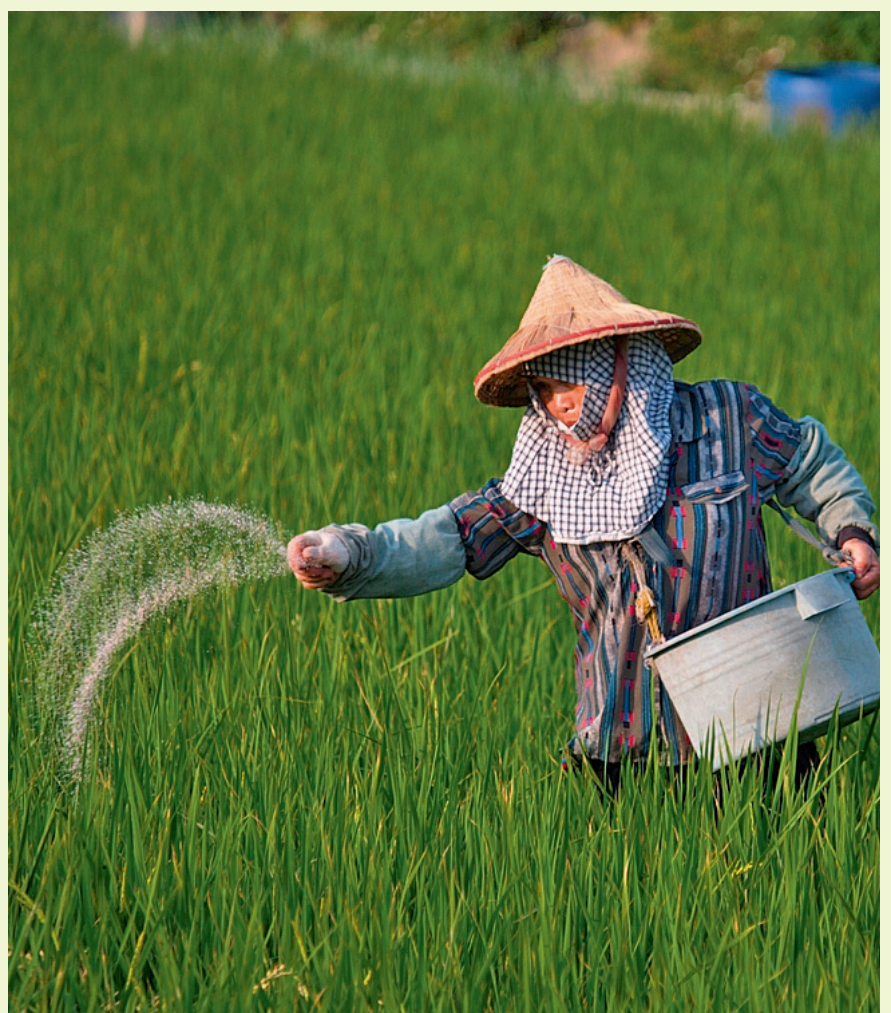

\section{Simplyscience.ch - die Website für Naturwissenschaften und Technik}

Geheimnisse des Alltags und wissenschaftliche Phänomene werden auf simplyscience.ch auf spannende Weise verständlich erklärt. Jugendliche finden spielend Zugang zu naturwissenschaftlichen und technischen Berufen oder können vor Ort an Veranstaltungen mehr über die Wunder der Wissenschaft erfahren. Ob interessante Storys, faszinierende Kolumnen, coole Quiz mit tollen Preisen oder ausführliche Hintergrundinformationen in spannenden Dossiers, auf simplyscience.ch ist man immer auf dem Laufenden und erfährt viel Neues über spannende Wissenschaftsbereiche. Für Lehrerinnen und Lehrer bietet sich an, simplyscience.ch im Schulunterricht einzubinden. Zum Beispiel ist die Kolumne zum Haber-Bosch-Verfahren ein Thema, das auch in der Schule aufgenommen wird. In einer spannenden Story verpackt, bietet dieses Thema sogar Anreiz zum Weiterlesen. Speziell für Lehrkräfte hat simplyscience.ch mit Kiknet.ch eine Unterrichtseinheit zum Thema Nanotechnologie bereitgestellt (http://www.kiknet.ch/partner/subsites/simply-science.html?L=0). Weitere Informationen: www. simplyscience.ch 